http://jmscr.igmpublication.org/home/ ISSN (e)-2347-176x ISSN (p) 2455-0450

crossref DOI: https://dx.doi.org/10.18535/jmscr/v9i6.03

\title{
Acral Lentiginous Melanoma in a 56 year old female
}

\author{
Authors \\ Dr Ritu Rani ${ }^{1}$, Dr Reena K Sharma ${ }^{2 *}$, Dr Mudita Gupta ${ }^{3}$ \\ ${ }^{1}$ Dermatologist, Civil Hospital Kangra, H.P. \\ ${ }^{2}$ Dermatologist, Civil Hospital Kangra \\ ${ }^{3}$ Assistant Professor, Department of Dermatology, IGMC, Shimla \\ *Corresponding Author \\ Dr Reena K Sharma \\ Dermatologist, Civil Hospital Kangra, HP, India
}

\begin{abstract}
Acral lentiginous melanoma present as darkly pigmented lesions over palms, soles and the nail. In Indian subcontinent, melanoma is rare as compared to western world due to skin type and topographical differences. The detailed clinical and histopathological examination helps to make early diagnosis and the timely interventions can be done. We hereby describe a case of 56 year old female diagnosed as ALM after a thorough workup.

Keywords: acral; melanoma; melanocytic nests.
\end{abstract}

\section{Introduction}

Acral lentiginous melanoma (ALM), is a subtype of cutaneous melanoma involving the acral parts. ${ }^{[1]}$ It was first defined by Reed as pigmented lesions occurring on the extremities. ${ }^{[2]}$ Clinically, it is characterized by irregular, black-brown, darkly pigmented, hyperkeratotic flat or verrucous lesions on the palms, soles of feet and the subungual regions of the nail. The diagnosis of ALM is challenging in the early stages of the disease. Delay in diagnosis and the aggressive nature can lead to poor patient outcome that highlight the need for early screening and detection.

\section{Case Report}

A 56 year old male presented with single painless, non itchy, dark colored lesion on right foot which he noticed 2 months ago and had increased in size till last month. On examination on the lateral border of planter aspect of right foot there was a single, irregular black-brown plaque of size $4 \times 3$ $\mathrm{cm}$ approximately with superficial ulceration with pigmented areas extending up to base of $5^{\text {th }}$ toe. The lymph node examination was normal Routine investigations including complete hemogram, LFTs, RFTs, were within normal limits. Histopathological examination shows a melanocytic proliferation at the dermoepidermal junction and epidermis. The melanocytes are arranged in nests and in solitary units within the epidermis and upper part of dermis. Occasional melanocytes can be seen in the lower epidermis. The neoplasm reaches both edges of the specimen. On the basis of clinical and histopathological examination the diagnosis of acral lentiginous 
melanoma was made. Patient was referred to surgery department where wide local excision was done under general anesthesia with $1 \mathrm{~cm}$ margins all around. Patient was followed up regularly every 3 months.

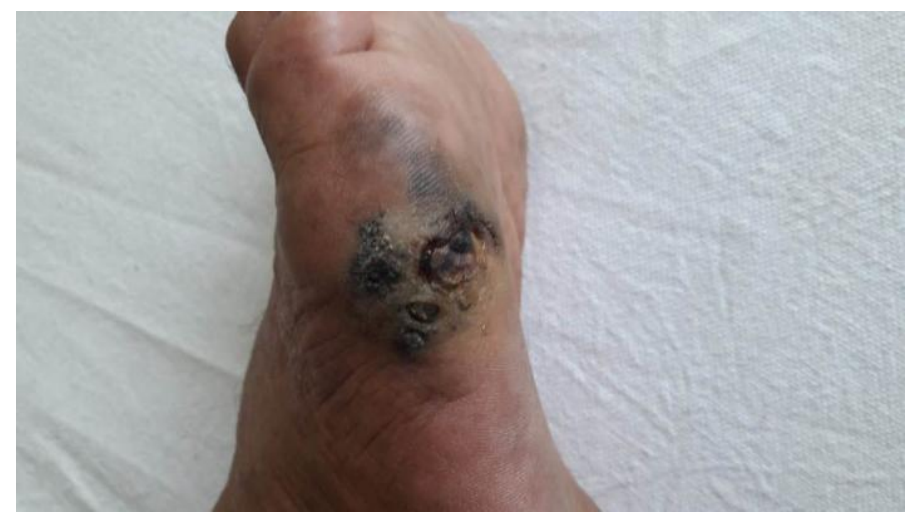

Figure 1. A single, irregular, dark brown-black plaque with superficial ulceration on the lateral border of right foot.

\section{Discussion}

Acral lentiginous melanoma (ALM) is an uncommon subtype of cutaneous melanoma that is characterized by pigmented lesions on palms, soles, or subungual region of nail. ${ }^{[3]}$ It accounts for approximately $5 \%$ of all melanomas. ${ }^{[4]}$ Melanomas usually occur on sun exposed areas but unlike other types, the role of UV-B in pathogenesis of ALM involving covered areas is not clear. ${ }^{[4]}$ However, studies suggest that trauma, stress, friction, maceration and previous penetrative injury to the sole may contribute to the melanomagenesis on the sole. Also, pre-existing skin cancer and malignant tumor history have significant association with ALM. ${ }^{[5,6]}$ Study done by Nagore et al. explained the role of tobacco smoking in melanoma especially in the patients who are over 60 years old. ${ }^{[7]}$

Clinically, ALM presents as a irregular, asymmetrical, brown-black, darkly pigmented macule or patch, on palms, soles or subungual areas of the nail. Ulceration is more commonly seen in ALM as compared to other subtypes of melanoma. ${ }^{[8]}$ ALM of the nail may present as longitudinal melanonychia or hyperpigmentation of nail folds, or hyponychium of the nail. It may present as amelanotic lesion simulating a pyogenic granuloma.

Histopathologically, the early stages of ALM may show subtle changes with scattered atypical melanocytes close to the basal layer. The radial growth phase is characterized by lentiginous proliferation of atypical melanocytes along the basal layer. ${ }^{[9]}$ There may be extension of atypical melanocytes along the sweat ducts into the dermis. The vertical growth phase often contains malignant melanocytes extending into the papillary dermis, with dysplasia of spindle cells, epidermal hyperplasia, and elongation of the rete ridges. ${ }^{[10]}$

Surgical excision remains the mainstay of treatment for most patients with ALM as in our case.

Treatment options for the advanced stage disease include BRAF inhibitors alone or in combination with MEK inhibitors such as vemurafenib and cobemetinib. ${ }^{[11]}$ Other treatment options include cKIT inhibitors like imatinib and cell death regulators targeting anti-CTLA4 and anti-PD1 antibodies. ${ }^{[11]}$ We present this case of ALM to highlight this rare variant of cutaneous melanoma. In Indian subcontinent, melanoma is rare as compared to western world due to skin type and topographical differences. However acral lentiginous melanoma is rare but reported from this region. Early diagnosis and treatment of ALM is necessary for better patient outcome.

\section{Conclusion}

The early diagnosis and treatment of ALM should be a priority. Also, all suspicious darkly pigmented lesions should be thoroughly investigated and timely interventions should be done for better patient outcomes.

\section{References}

1. Asgari, M. M., Shen, L., Sokil, M. M., Yeh, I. \& Jorgenson, E. Prognostic factors and survival in acral lentiginous melanoma. Br. J. Dermatol. 177, 428-435 (2017). 
2. Reed, R. J. New Concepts in Surgical Pathology of the Skin. (John Wiley \& Sons, 1976).

3. Ward WH, Lambreton F, Goel N, Yu J, Farma J: Clinical presentation and staging of melanoma . Clin Melanoma Etiol Ther. 2017, 21:6.

4. Garbe C, Bauer J: Types of Primary Melanomas. Dermatology (4th ed). Bolognia J (ed): Elsevier, Philadelphia, PA; 2018.

5. Green A, McCredie M, MacKie R, Giles $\mathrm{G}$, Young P, Morton C, et al. A casecontrol study of melanomas of the soles and palms (Australia and Scotland). Cancer Causes Control. 1999;10(1):21-5.

6. Dediol I, Bulat V, Zivkovic MV, Markovic BM, Situm M. Dysplastic nevus--risk factor or disguise for melanoma. Coll Antropol. 2011;35 Suppl 2:311-3.

7. Nagore E, Hueso L, Botella-Estrada R, Alfaro-Rubio A, Serna I, Guallar J, et al. Smoking, sun exposure, number of nevi and previous neoplasias are risk factors for melanoma in older patients (60 years and over). J Eur Acad Dermatol Venereol. 2010;24(1):50-7.

8. Badge SA, Meshram A, Ovhal A. Acral lentiginous melanoma. Med J Dr. D.Y. Patil Univ. 2015;8(4).

9. Kuchelmeister C, Schaumburg-Lever G, Garbe C. Acral cutaneous melanoma in caucasians: Clinical features, histopathology and prognosis in 112 patients. Br J Dermatol. 2000;143(2):27580.

10. Krementz ET, Reed RJ, Coleman WP, Sutherland CM, Carter RD, Campbell M: Acral lentiginous melanoma. Ann Surg. 1982, 195:632-645.

11. Leonardi GC, Falzone L, Salemi R, et al.: Cutaneous melanoma: from pathogenesis to therapy (Review) . Int J Oncol. 2018, 52:1071-1080. 